\title{
Survival Benefit of Intervention Treatment in Advanced Anaplastic Thyroid Cancer
}

\author{
Pornthep Kasemsiri (iD, ${ }^{1,2}$ Pimpika Chaisakgreenon ${ }^{(D)},{ }^{1}$ Patravoot Vatanasapt $\left(\mathbb{D},{ }^{1,2}\right.$ \\ Supawan Laohasiriwong $\mathbb{D}^{\mathrm{D}},{ }^{1,2}$ Watchareeporn Teeramatwanich $\mathbb{D}^{\mathrm{D}},{ }^{1,2}$ \\ Cattleya Thongrong $\mathbb{D}^{2,3}$ Teeraporn Ratanaanekchai $\mathbb{D}^{1,2}$ and Surapol Suetrong $\mathbb{D}^{1}$ \\ ${ }^{1}$ Department of Otorhinolaryngology, Srinagarind Hospital, Faculty of Medicine, Khon Kaen University, Khon Kaen, Thailand \\ ${ }^{2}$ Khon Kaen Head and Neck Oncology Research, Khon Kaen, Thailand \\ ${ }^{3}$ Department of Anesthesiology, Srinagarind Hospital, Faculty of Medicine, Khon Kaen University, Khon Kaen, Thailand
}

Correspondence should be addressed to Pornthep Kasemsiri; pkcolumbus99@gmail.com

Received 17 January 2021; Revised 19 May 2021; Accepted 28 May 2021; Published 3 June 2021

Academic Editor: Gaetano Gallo

Copyright (c) 2021 Pornthep Kasemsiri et al. This is an open access article distributed under the Creative Commons Attribution License, which permits unrestricted use, distribution, and reproduction in any medium, provided the original work is properly cited.

\begin{abstract}
Background. The management of anaplastic thyroid cancer (ATC) is controversial; thus, proper treatment and prognostic factors should be investigated. Objectives. To compare the survival outcomes of the intervention and palliative treatment in ATC patients. Methods. A hospital-based retrospective study was conducted at a single tertiary university hospital. The medical record charts were retrieved from November 20, 1987, to December 31, 2016. The final follow-up ended by December 31, 2017. The patients' demographic data, laboratory data, clinical presentation, and treatment modality results were analyzed. Results. One hundred twenty-one records were analyzed with a one-year overall survival rate of 3.5\% (median survival time: 77 days); however, 16 cases had insufficient data to classify staging and treatment modalities. Therefore, 105 ATC patients (37 with stage IVa, 39 with stage IVb, and 29 with stage IVc disease) were included with a one-year overall survival rate of $4.0 \%$ (median survival time of 82 days). Intervention treatment allowed longer median survival times $(p<0.05)$ and a better survival rate $(p<0.05)$. Among the interventional treatment groups, postoperative chemoradiation yielded the longest median survival time (187 days) and the highest survival rate $(20 \%)(p<0.05)$. The intervention modality allowed a better median survival time at all stages, particularly in stage IVa $(p<0.05)$. Unfavorable prognostic factors were adjusted for in a multiple Cox regression model showing that significant factors included age $\geq 65$ years (hazard ratio HR: 2.57 ), palliative treatment (HR: 1.85 ), and leukocytosis $\geq 10,000$ cells $/ \mathrm{mm}^{3}$ (HR: 2.76). Conclusions. Intervention treatment provided a better survival outcome in all stages, particularly in stage IVa, with a significantly better median survival time. Among interventional treatments, postoperative chemoradiation led to the longest survival rate, suggesting that this treatment should be considered in ATC patients with resectable tumors and no poor prognostic factors, such as older age and leukocytosis.
\end{abstract}

\section{Introduction}

Anaplastic thyroid cancer (ATC) is a rare disease. Although ATC only accounts for $1 \%-2 \%$ of all thyroid malignancies, it is a rapidly growing tumor with extremely aggressive behavior, accounting for more than $50 \%$ of all thyroid-related mortality [1-3]. Several studies have reported a median overall survival rate of less than 6 months and a 1-year survival rate of $20 \%$ [3-5]. Regarding ATC treatment, multimodality (including surgery, radiotherapy, and systemic therapy) is required for improved survival rates. The complete surgical removal of tumors in ATC is a good option for limited tumor invasion; however, most patients present with a rapidly enlarging mass [6-8]. Additionally, up to $70 \%$ of patients were reported to have aggressive ATC with invasion into surrounding tissues, including the muscle (65\%), trachea (46\%), esophagus (44\%), and larynx (13\%) [1]. Therefore, other interventional treatments were 
introduced to combine multimodality treatment to combat aggressive ATC. Sugitani et al. [9] reported that surgery and external beam radiotherapy $\geq 40 \mathrm{~Gy}$ were predictors of significantly better overall survival in any stage of ATC. Regarding systemic therapy, chemotherapy has been increasingly used over the last few decades [10]. Sugitani et al. [9] showed that chemotherapy was a predictor of significantly better overall survival for patients with stage IVB or IVC disease. Furthermore, novel systemic therapy (bovine serum ribonuclease [11], bone morphogenic protein $[12]$, and p53 gene therapy $[13,14])$ was proposed to alter the course of ATC. Therefore, the combination of multimodality treatment seemed to allow improvement in survival outcomes. However, these interventional treatments do not achieve universally beneficial outcomes; conversely, adverse side effects from interventional treatments may worsen the outcomes and make the poor survival rate even worse in patients with compromised health status. Therefore, interventional treatment should be reserved for patients with a good health status who can tolerate treatment side effects. For patients with a poor health status, supportive or palliative treatment should be considered to improve the quality of life and avoid the side effects of interventional treatments. However, survival rate data in patients with palliative treatment are lacking, as is comparative data assessing palliative care outcomes against the benefits of interventional treatments. Therefore, this study aimed to compare the survival outcomes from palliative versus intervention care and investigate unfavorable prognostic factors predictive of poor survival outcomes.

\section{Materials and Methods}

2.1. Study Design and Data Collection. A hospital-based retrospective study was conducted with anaplastic thyroid cancer patients at a single tertiary university hospital. The medical record charts from the ATC patients from November 20, 1987, to December 31, 2016, were retrieved. ATC was diagnosed based on fine-needle aspiration cytology and/ or histopathology from the biopsy or surgical specimen. The patients' demographic data, laboratory data, clinical presentation, and treatment modality results were assessed. For the staging of ATC, we used the standard TNM classification of the 8th edition AJCC staging system. Regarding our treatment modality, total thyroidectomy was performed in patients with tumors localized at the thyroid gland, whereas thyroidectomy with extensive resection of the surrounding tissue was performed for patients with resectable extrathyroid invasion. Neck dissection at levels II to VI was performed in patients with clinical or cytopathological lymph nodes, while neck dissection at level VI was performed in patients with clinically negative cervical lymph nodes. Other modalities of treatment and radiotherapy were classified according to the total radiation dose. We allocated patients who received doses of more than $40 \mathrm{~Gy}$ to the intervention group and those with doses of less than $40 \mathrm{~Gy}$ to the palliative group. For the chemotherapy modality, we classified chemotherapy plus other therapy modalities (surgery and/or radiotherapy) as an intervention group, whereas a single chemotherapy modality was defined as the palliative group. The palliative group was reserved for patients with a tumor that was beyond surgery and poor health status and who were not candidates for definite radiotherapy. The follow-up time started from the date of the first treatment and ended by December 31, 2017.

2.2. Statistical Analysis. Statistical analysis was performed using STATA (v 10.0; Stata Corp., Texas, USA). Survival duration was analyzed using the days from the date of diagnosis to the date of death. Kaplan-Meier analysis was used to demonstrate the survival curve. Patients who were lost to follow-up or survived were considered censors. A comparison of the survival curves between the intervention and palliative group was performed using the log-rank test in each stage. Furthermore, univariate analysis was used for the Cox proportional hazard regression to identify significant prognostic factors. After that, the statistical significance of covariates on survival was adjusted with multiple Cox regression analysis to identify independent prognostic factors. $p<0.05$ was considered statistically significant.

2.3. Ethics. The study was approved by the local ethics research committee (HE611221).

\section{Results}

3.1. Patient Demographics. The data for 121 patients with ATC (42 men and 79 women) were retrieved from the hospital database (Table 1). Almost half of the patients $(40.5 \%)$ were in the 61 - to 70 -year age range. Most patients presented a tumor $\geq 5 \mathrm{~cm}(72.7 \%)$, and $11.6 \%$ showed extrathyroid invasion of vital structures, including the carotid sheath, subclavian artery, and intrathoracic structures.

3.2. Survival Data. The one-year overall survival rate of $3.5 \%$ (median survival time: 77 days (95\% CI: 57-88)) with a median follow-up time of 74 days (range: 5-4,061 days) was observed in our 121 patients (Figure 1(a)); however, 16 ATC patients had insufficient data to classify TNM staging and treatment modalities. The remaining 105 ATC patients were classified as follows: stage IVa, 37 patients; IVb, 39 patients; IVc, 29 patients. The common pattern of regional cervical lymph node metastasis was a unilateral single node (19.8\%), and the most common site of distant metastasis was the lung (22.6\%). Regarding treatment modality, 49 ATC patients had received palliative treatment $(35.2 \%$ supportive treatment and $11.4 \%$ palliative radiation), while 56 ATC patients received interventional treatment, including surgery alone $(27.6 \%)$, chemoradiation $(8.5 \%)$, surgery combined radiation $(12.4 \%)$, and surgery combined chemoradiation $(4.7 \%)$. The overall survival rate was $4.0 \%$ at 1 year (median survival time of 82 days (95\% CI: 63-96)) in the 105 patients (Figure 1(b)). Comparing the interventional and palliative treatments, the overall median survival time of the interventional treatment (110 days) was almost twice as long and was significantly different (log-rank test; $p<0.05$; 
TABLe 1: Demographic data.

\begin{tabular}{|c|c|}
\hline Characteristic & $N(\%)$ \\
\hline \multicolumn{2}{|l|}{ Gender } \\
\hline Female & $79(65.3)$ \\
\hline Male & $42(34.7)$ \\
\hline \multicolumn{2}{|l|}{ Age (years) } \\
\hline$\leq 40$ & $3(2.5)$ \\
\hline $41-50$ & $6(4.9)$ \\
\hline $51-60$ & $23(19.0)$ \\
\hline $61-70$ & $49(40.5)$ \\
\hline$\geq 70$ & $40(33.1)$ \\
\hline \multicolumn{2}{|l|}{ Underlying disease } \\
\hline No/Unknown & $88(72.7)$ \\
\hline Diabetes mellitus & $19(15.7)$ \\
\hline Hypertension & $17(14.1)$ \\
\hline Dyslipidemia & $3(2.5)$ \\
\hline Other & $15(12.4)$ \\
\hline \multicolumn{2}{|l|}{ Thyroid function test } \\
\hline Hypothyroid & $8(6.6)$ \\
\hline Euthyroid & $17(14.1)$ \\
\hline Hyperthyroid & $3(2.5)$ \\
\hline Unknown & $93(76.9)$ \\
\hline \multicolumn{2}{|l|}{ WBC (cells/ml ${ }^{3}$ ) } \\
\hline$\geq 10,000$ & $48(39.7)$ \\
\hline$<10,000$ & $31(25.6)$ \\
\hline Unknown & $42(34.7)$ \\
\hline \multicolumn{2}{|l|}{ Tumor size $(\mathrm{cm})$} \\
\hline$<5$ & $6(4.9)$ \\
\hline$\geq 5$ & $88(72.7)$ \\
\hline Unknown & $27(22.3)$ \\
\hline Extrathyroid invasion involved vital structures & $14(11.6)$ \\
\hline \multicolumn{2}{|l|}{ Cervical lymph node metastasis } \\
\hline No/Unknown & $69(57.0)$ \\
\hline Unilateral single & $24(19.8)$ \\
\hline Unilateral multiple & $18(14.9)$ \\
\hline Bilateral & $10(8.3)$ \\
\hline \multicolumn{2}{|l|}{ Distance metastasis } \\
\hline No/Unknown & $89(71.8)$ \\
\hline Lung & $28(22.6)$ \\
\hline Bone & $5(4.0)$ \\
\hline Liver & $2(1.6)$ \\
\hline \multicolumn{2}{|l|}{ Staging } \\
\hline IVa & $37(30.6)$ \\
\hline $\mathrm{IVb}$ & $39(32.2)$ \\
\hline IVc & $29(23.9)$ \\
\hline Unknown & $16(13.2)$ \\
\hline \multicolumn{2}{|l|}{ Treatment } \\
\hline Supportive treatment & $37(30.6)$ \\
\hline Palliative radiation & $12(9.9)$ \\
\hline Surgery alone & $29(23.9)$ \\
\hline Chemoradiation & $9(7.4)$ \\
\hline Surgery combined radiation & $13(10.7)$ \\
\hline Surgery combined chemoradiation & $5(4.1)$ \\
\hline Unknown & $16(13.2)$ \\
\hline
\end{tabular}

Figure 2(a)) from that of the palliative treatment group (58 days). Among the interventional treatment groups, surgery with postoperative chemoradiation yielded the longest median survival time of 187 days and the longest survival rate of $20 \%$ (log-rank test; $p<0.05$ ) (Figure $2(b)$ ). The median survival time of intervention and palliative treatment was also compared in each stage. In stage IVa, the interventional treatment group (118 days (95\% CI: 54-160)) had significantly longer survival than the palliative treatment group (33 days (95\% CI: 10-46)) ( $p \leq 0.001$; Figure 3(a)); however, the median survival time of the interventional treatment group was not significantly longer than that of the palliative treatment group in stages IVb (intervention: 110 days (95\% CI: 64-177); palliative: 63 days (95\% CI: 49-133); $p=0.63$; Figure 3(b)) and IVc (intervention: 96 days $(95 \%$ CI: 10-168); palliative: 64 days (95\% CI: 37-93); $p=0.06$; Figure 3(c)).

3.3. Analysis of Factors Affecting Prognosis. Regarding prognostic factors, the univariate analysis found significantly poorer outcomes associated with an age $\geq 65$ years (hazard ratio (HR): 1.6), palliative treatment (HR: 2.0), hypothyroidism (HR: 4.5), and leukocytosis (HR: 2.1) (Table 2). After that, these variables were adjusted for in the multivariate analysis, and an age $\geq 65$ years (HR: 2.6), palliative treatment (HR: 1.9), and leukocytosis (HR: 2.8) were demonstrated to be significant independent variables for poorer outcomes (Table 3).

\section{Discussion}

Our results showed that most ATC patients were older than 60 years $(73.6 \%)$ with a male to female ratio of $1: 1.9$, very similar to previous findings [15-17]. However, most of our ATC patients $(72.7 \%)$ presented with tumors $\geq 5 \mathrm{~cm}$ in diameter, slightly larger than those reported in previous studies $(53.0 \%-68.9 \%)[15,18]$. According to TNM staging, our patients were approximately equally distributed across each stage (35.2\% for IVa, 37.1\% for IVb, and $27.6 \%$ for IVc). In stage IVb and IVc cases with extrathyroid invasion, the tissue surrounding the thyroid gland was frequently involved, making complete removal challenging in patients with extensive involvement of vital structures. We found that $11.6 \%$ of our patients presented with tumors involving vital structures. Distant metastasis was also a prognostic factor for a poor survival outcome in $22.6 \%$ of patients in our series. Previous research reported ATC survival outcomes ranging from 2 to 10 months and $>2$-year survival rates of $0 \%-10 \%$ [10], which were similar to our findings of the median survival time and 1-year survival rate of 77-82 days and $3.5 \%-4.0 \%$, respectively. Survival outcomes in prior retrospective studies vary depending on the sample size, baseline demographic data, and selection bias. Our survival outcomes were likely worse than those of previous studies because of numerous cases with a large tumor involving vital structures and distant metastasis. Treatment modality has important effects on survival outcomes. Some studies advocate that multimodality treatment has benefits [19-21]; however, few studies report significant survival benefits from multimodality treatment $[22,23]$. Our study found that interventional treatments provided better survival outcomes than palliative treatment $(p<0.05)$ in overall staging. However, the surgery and postoperative chemoradiation 


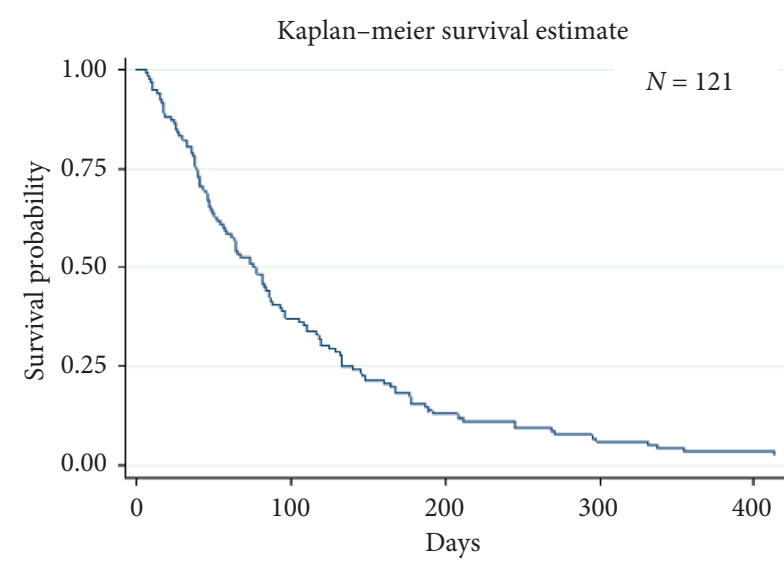

(a)

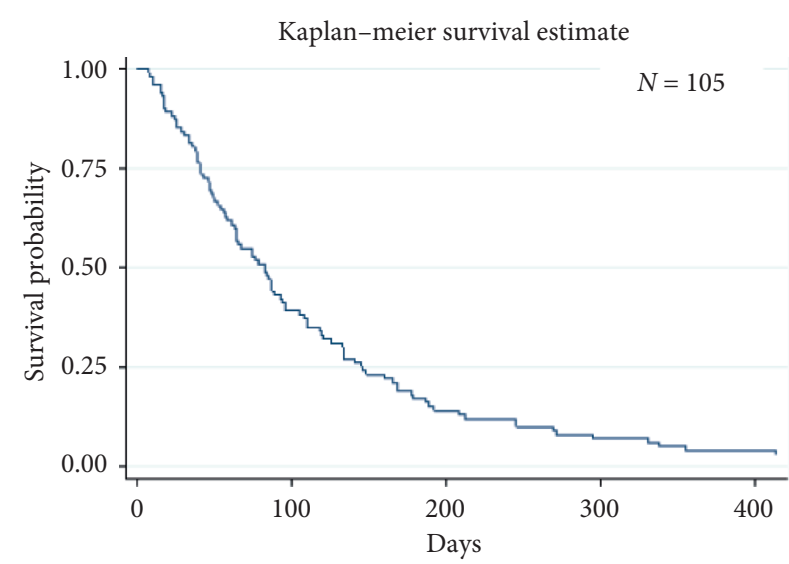

(b)

FIgURE 1: The 1-year overall survival rate and median survival time of all ATC patients were 3.5\% (95\% CI: 1.7-8.0) and 77 days (95\% CI: 57-88), respectively (a). However, 16 ATC patients had insufficient data to classify the staging and modality of treatment. Thus, 105 ATC patients showed a 1-year overall survival rate of $4 \%$ (95\% CI: 1.3-9.2) and a median survival time of 82 days (95\% CI: 63-96) (b).

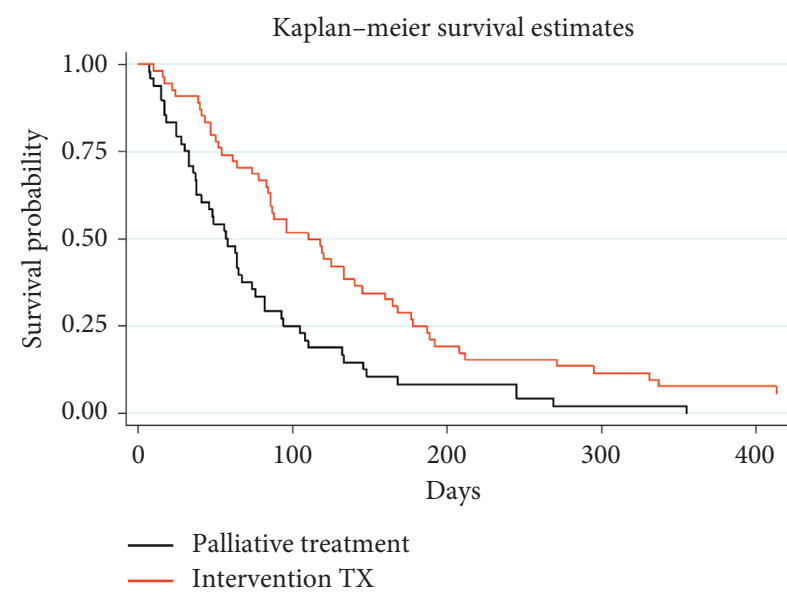

(a)

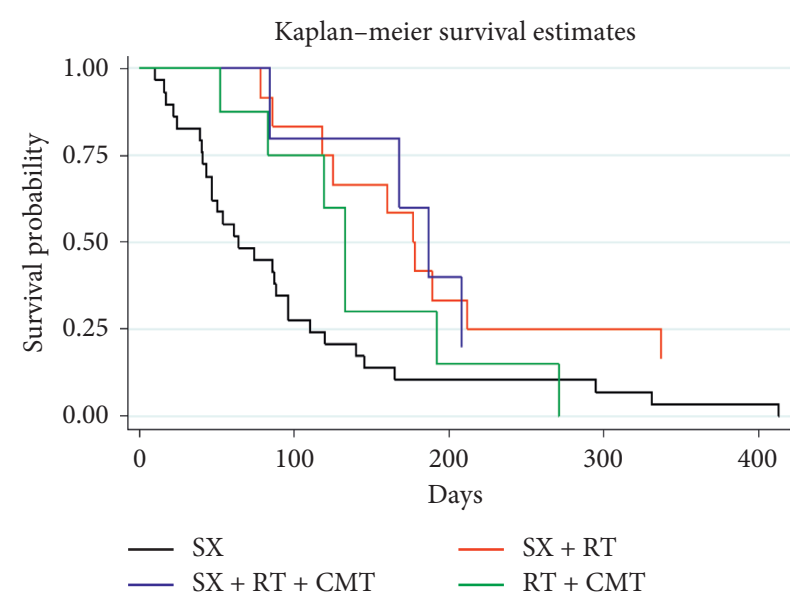

(b)

Figure 2: One hundred five ATC patients were classified, among whom 49 received palliative modality and 56 patients received interventional treatment. Intervention treatment allowed a median survival time of 110 days (95\% CI: 84-140) that was better than palliative treatment. Palliative treatment allowed a median survival time of 58 days (95\% CI: 38-74). Furthermore, the Kaplan-Meier survival curve was analyzed using the log-rank test, revealing that interventional treatment was significantly better than palliative treatment $(p=0.0006)$ (a). In the interventional treatment, the combination of surgery with postoperative chemoradiation showed the best survival rate (log-rank test; $p=0.01)$. The median survival time for interventional modalities was analyzed by subgroup and showed times of 187 days $(95 \%$ CI: 84-208) in the surgery combined with postoperative chemoradiation treatment, 177 days (95\% CI: 86-337) in the surgery combined with radiation treatment, 133 days (95\% CI: 52-192) in the chemoradiation treatment, and 64 days (95\% CI: 43-96) in the surgery-alone treatment (b).

combination provided the best 1-year survival rate of $20.0 \%$ among the interventional treatment groups. These findings compare well with a previous study [21] that showed that complete ATC resection combined with postoperative adjuvant chemotherapy and irradiation resulted in longer-term survival, even with persistent minimal disease. Although interventional treatment seemed to provide superior survival outcome benefits, we also investigated possible differential effects across different staging levels. We found that intervention provided significantly better outcomes than palliative care in stage IVa $(p<0.05)$. Intervention treatment was also better than palliative care in stages IVb and IVc $(p>0.05)$ but not at a statistically significant level, possibly because of more aggressive tumors in these advanced stages.

Age, gender, tumor size, the extent of disease at presentation, acute symptoms, distant metastasis, leukocytosis, and multimodality therapy are previously reported prognostic variables associated with survival outcome $[6,7,20,21,24-26]$. In our study, the ATC patient prognosis mainly depended on age, leukocytosis, and treatment. Glaser et al. [27] reported that an age $\geq 65$ years was an unfavorable prognostic factor. This finding was similar to our study finding that showed that older age was a factor for 


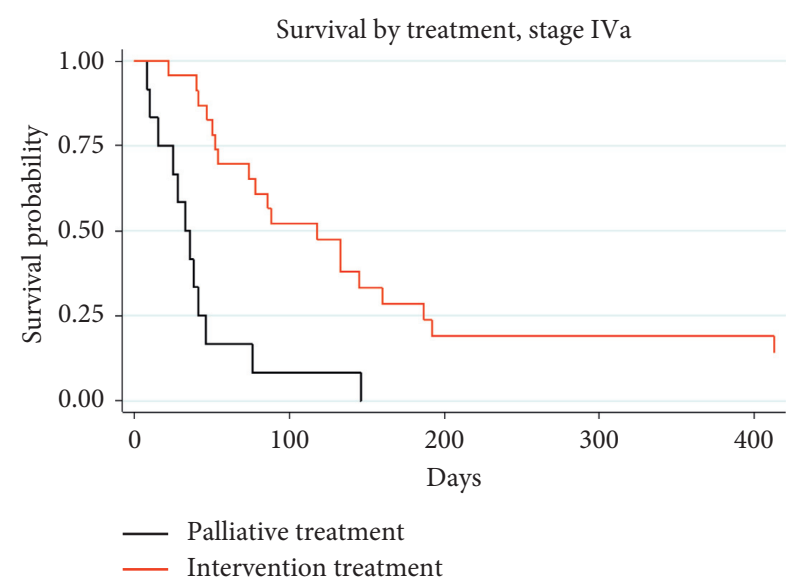

(a)

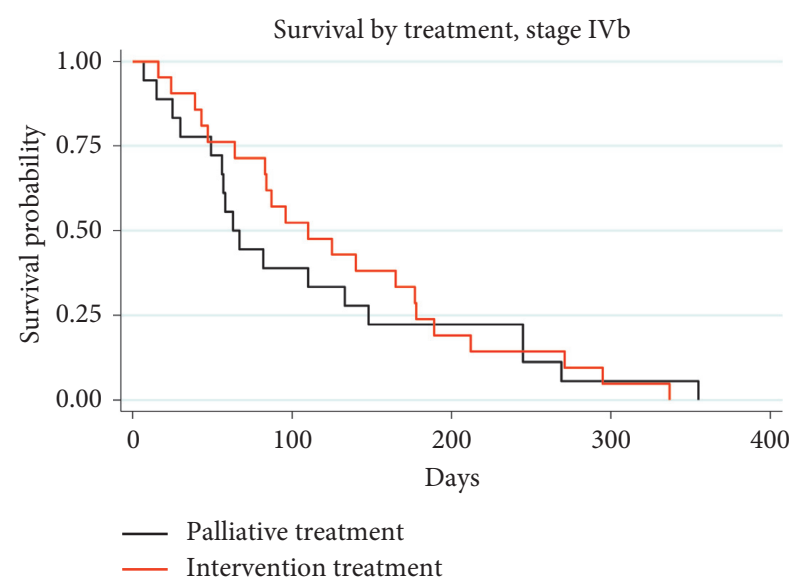

(b)

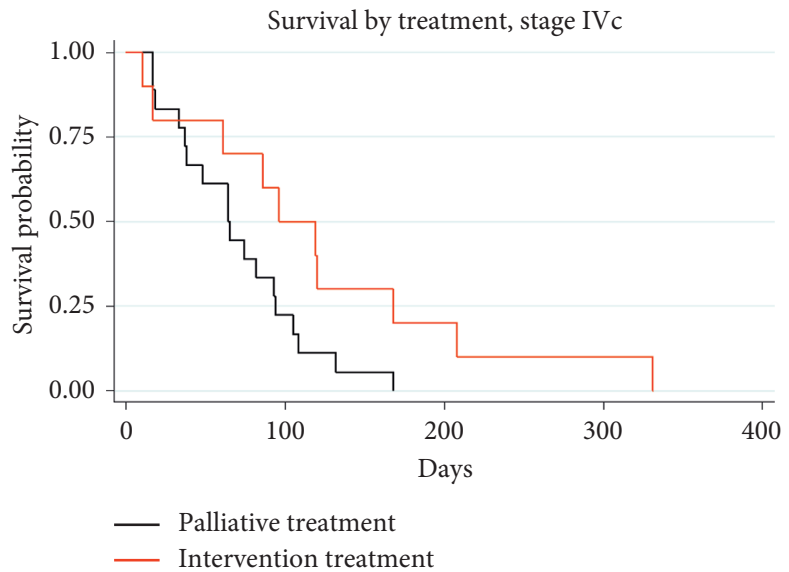

(c)

Figure 3: Thirty-seven ATC patients in stage IVa had a median survival time of 118 days (95\% CI: 54-160) in the interventional treatment group, whereas the palliative treatment group had a median survival time of 33 days (95\% CI: 10-46). This difference was statistically significant $(p \leq 0.001)$. The Kaplan-Meier curve showed the benefit survival rate in the intervention group (log-rank test; $p \leq 0.001)$ (a). In 39 patients with ATC stage IVb, the interventional treatment allowed a median survival time of 110 days (95\% CI: 64-177), which was better than palliative treatment (median survival time: 63 days (95\% CI: 49-133)); however, the median time survival difference was not statistically significant $(p=0.63)$. The Kaplan-Meier curve showed that the intervention group seemed superior to palliative treatment but did not reach statistical significance (log-rank test; $p=0.67)(b)$. Regarding the median survival time of 29 patients with ATC stage IVc, the intervention group (96 days (95\% CI: 10-168)) was not significantly better than the palliative group (64 days (95\% CI: $37-93))(p=0.06)$. However, the Kaplan-Meier survival curve of the intervention group was not significantly better than that of the palliative group (log-rank test; $p=0.055)(\mathrm{c})$.

significantly higher mortality (HR: 1.55). Other authors have also reported that older age was a poor prognostic factor, but old age was variously defined. The range for old age was reported as $\geq 60-75$ years in previous studies [25, 28, 29]. Furthermore, leukocytosis was observed to also predict poor survival outcomes. Jiang et al. [15] and Sugitani et al. [9] found HRs of 1.12 and 1.48, respectively. In our series, a white blood cell count $\geq 10,000 / \mathrm{ml}^{3}$ had a hazard ratio of 2.76 $(p<0.001)$ from the Cox regression analysis. This finding was comparable with that in previous reports investigating the effects of leukemoid paraneoplastic reaction by ATC tumor-secreted cytokines, including granulocyte-colony stimulating factor, granulocyte macrophage-CSF, and interleukin-6 [30, 31]. The final significant prognostic factor found in our study, treatment modality, revealed that palliative treatment predicted the poorest overall survival outcome, with an HR of $1.85(p<0.05)$. However, selection bias makes this finding unsurprising given that palliative care patients usually have advanced disease with high mortality.

Sugitani et al. [9] classified the modality benefits in each ATC staging and found that postoperative chemoradiation was a significantly favorable prognostic factor in stage IVb (HR: $0.45 ; p=0.083$ ); however, in stage IVa, its benefits did not reach a statistically significant level (HR: $0.21 ; p=0.19$ ). Although controversy persists concerning proper ATC treatment protocols, several previous studies suggest that multimodal treatment allows a longer survival rate. Kobayashi et al. [24] suggested active multimodality treatment at the early stage. The multimodality protocol of surgery and chemoradiation has been advocated as offering the highest survival rate [32-34]. In the present study, the 
TABLE 2: Unadjusted univariable Cox proportional hazard model of prognostic factors.

\begin{tabular}{|c|c|c|}
\hline Variable & Hazard ratio $(95 \% \mathrm{CI})$ & $p$ value \\
\hline $\begin{array}{l}\text { Age (years) } \\
\quad<65 \\
\geq 65\end{array}$ & $\begin{array}{l}\text { Reference } \\
1.6(1.1-2.0)\end{array}$ & 0.022 \\
\hline $\begin{array}{l}\text { Treatment } \\
\quad \text { Intervention } \\
\text { Palliative } \\
\end{array}$ & $\begin{array}{c}\text { Reference } \\
2.0(1.3-3.0)\end{array}$ & 0.001 \\
\hline $\begin{array}{l}\text { Staging } \\
4 \mathrm{a} \\
4 \mathrm{~b} \\
4 \mathrm{c} \\
\end{array}$ & $\begin{array}{c}\text { Reference } \\
0.95(0.7-1.7) \\
1.35(0.92-2.27) \\
\end{array}$ & $\begin{array}{l}0.819 \\
0.221 \\
\end{array}$ \\
\hline $\begin{array}{l}\text { Thyroid function test } \\
\text { Euthyroid } \\
\text { Hypothyroid } \\
\text { Hyperthyroid } \\
\end{array}$ & $\begin{array}{c}\text { Reference } \\
4.50(1.19-13.57) \\
3.75(0.93-15.07) \\
\end{array}$ & $\begin{array}{l}0.008 \\
0.063\end{array}$ \\
\hline $\begin{array}{l}\left.\text { White blood cell (cells } / \mathrm{ml}^{3}\right) \\
\quad<10000 \\
\geq 10000\end{array}$ & $\begin{array}{c}\text { Reference } \\
2.05(1.25-3.35)\end{array}$ & 0.004 \\
\hline $\begin{array}{l}\text { Underlying disease } \\
\text { Absent } \\
\text { Present }\end{array}$ & $\begin{array}{c}\text { Reference } \\
1.19(0.79-1.81)\end{array}$ & 0.403 \\
\hline $\begin{array}{l}\text { Tumor size }(\mathrm{cm}) \\
\quad<5 \\
\quad \geq 5\end{array}$ & $\begin{array}{c}\text { Reference } \\
2.32(0.84-6.38)\end{array}$ & 0.104 \\
\hline $\begin{array}{l}\text { Extrathyroid extension } \\
\text { No } \\
\text { Yes }\end{array}$ & $\begin{array}{c}\text { Reference } \\
1.12(0.75-1.69)\end{array}$ & 0.569 \\
\hline $\begin{array}{l}\text { Cervical lymph node metastasis } \\
\text { No } \\
\text { Unilateral single } \\
\text { Unilateral multiple } \\
\text { Bilateral }\end{array}$ & $\begin{array}{c}\text { Reference } \\
1.03(0.64-1.65) \\
0.76(0.44-1.31) \\
1.41(0.71-2.77)\end{array}$ & $\begin{array}{l}0.914 \\
0.328 \\
0.323\end{array}$ \\
\hline $\begin{array}{l}\text { Distance metastasis } \\
\text { No } \\
\text { Present (lung, bone, and liver) }\end{array}$ & $\begin{array}{c}\text { Reference } \\
1.33\end{array}$ & 0.183 \\
\hline
\end{tabular}

TABle 3: Adjusted multivariable Cox proportional hazard models of prognostic factors.

\begin{tabular}{lcc}
\hline Variable & $\begin{array}{c}\text { Adjust hazard ratio }(95 \% \\
\text { CI) }\end{array}$ & $p$ value \\
\hline $\begin{array}{l}\text { Age (years) } \\
<65\end{array}$ & Reference & \\
$\geq 65$ & $2.6(1.5-4.4)$ & 0.001 \\
\hline $\begin{array}{l}\text { Treatment } \\
\quad \text { Intervention }\end{array}$ & \\
$\quad$ Ralliation & $1.9(1.1-3.1)$ & 0.016 \\
\hline $\begin{array}{l}\text { White blood cell }\left(\text { cells } / \mathrm{ml}^{3}\right) \\
\quad<10000\end{array}$ & Reference & \\
$\geq 10000$ & $2.8(1.6-4.9)$ & $<0.001$ \\
\hline
\end{tabular}

combined modality of postoperative chemoradiation and radiotherapy led to longer median survival times of 187 days and 177 days, respectively, than surgery alone, which led to a survival rate of 64 days, again supporting the advantage of multimodality treatment in improving the survival outcomes. A negative prognostic association has been reported for hypothyroidism. Our study found that hypothyroidism was a negative predictor in the univariate analysis; however, the multivariate regression analysis showed that this difference was not statistically significant. Jiang et al. [15] found similar findings where the serum T4 levels were not statistically significant in Cox regression analysis. However, they observed that patients with low T4 levels had significantly lower survival rates than those with normal T4 levels. Several authors proposed that hypothyroidism may occur due to the tumor damaging the normal thyroid tissue $[35,36]$ and inhibition of changes in T4-to-T4 binding globulin by unsaturated fatty acids from hypoxic or injured tissue in severely ill patients [37]. Therefore, low T4 levels may represent a late stage of ATC with severe disease that indicates poor survival outcomes.

ATC is an extremely aggressive and rapidly progressing tumor that makes it difficult to use a randomized prospective protocol to evaluate treatment and survival outcomes; therefore, a retrospective chart review was selected as a feasible approach for this study. Although our study includes the limitations of retrospective studies, it showed that a multimodality treatment was superior to a palliative modality, particularly the combination of surgery and chemoradiation. Furthermore, we found that not only palliative treatment but also age and leukocytosis were unfavorable prognostic factors for predicting mortality outcomes. In the future, more laboratory information and detailed clinical data would allow for better investigation of prognostic factors.

\section{Conclusion}

The results obtained from the current study showed that interventional treatment led to better survival outcomes in all stages of ATC, particularly in stage IVa. Among interventional treatments, postoperative chemoradiation led to the longest survival rate and should be considered for ATC patients with a resectable tumor and no poor prognostic factors. Factors (including older age $\geq 65$ years, leukocytosis $\geq 10,000 \mathrm{cells} / \mathrm{ml}^{3}$, and palliative treatment) should be considered as unfavorable predictive prognostic factors that may help to decide on the management of ATC.

\section{Abbreviations}

ATC: Anaplastic thyroid cancer

AJCC: American Joint Committee on Cancer

HR: Hazard ratio.

\section{Data Availability}

The data that support the findings of this study are available from the corresponding author upon reasonable request.

\section{Disclosure}

The study has been presented as a preprint in the following link: http://www.researchsqurare.com/article/rs-120518/v1. 


\section{Conflicts of Interest}

The authors declare that there are no conflicts of interest regarding the publication of this paper.

\section{Acknowledgments}

The authors thank Prof. John F. Smith under the aegis of the Publication Clinic, Khon Kaen University, for assistance with the English-language presentation, and Chalongpol Santhong for assistance with the data analysis. This study was funded by the Faculty of Medicine, Khon Kaen University, Thailand (Grant no. MN61204).

\section{References}

[1] R. C. Smallridge, K. B. Ain, S. L. Asa et al., "American Thyroid Association guidelines for management of patients with anaplastic thyroid cancer," Thyroid, vol. 22, no. 11, pp. 1104-1139, 2012.

[2] D. Giuffrida and H. Gharib, "Anaplastic thyroid carcinoma: current diagnosis and treatment," Annals of Oncology, vol. 11, no. 9, pp. 1083-1090, 2000.

[3] A. Taccaliti, F. Silvetti, G. Palmonella, and M. Boscaro, "Anaplastic thyroid carcinoma," Frontiers in Endocrinology, vol. 3, p. 84, 2012.

[4] S. M. Lim, S.-J. Shin, W. Y. Chung et al., "Treatment outcome of patients with anaplastic thyroid cancer: a single center experience," Yonsei Medical Journal, vol. 53, no. 2, pp. 352-357, 2012.

[5] E. Kebebew, F. S. Greenspan, O. H. Clark, K. A. Woeber, and A. McMillan, "Anaplastic thyroid carcinoma," Cancer, vol. 103, no. 7, pp. 1330-1335, 2005.

[6] C. J. C. Nel, J. A. van Heerden, J. R. Goellner et al., "Anaplastic carcinoma of the thyroid: a clinicopathologic study of 82 cases," Mayo Clinic Proceedings, vol. 60, no. 1, pp. 51-58, 1985.

[7] Y.S. S. Venkatesh, N. G. Ordonez, P. N. Schultz, R. C. Hickey, H. Goepfert, and N. A. Samaan, "Anaplastic carcinoma of the thyroid: a clinicopathologic study of 121 cases," Cancer, vol. 66, no. 2, pp. 321-330, 1990.

[8] T. Hadar, C. Mor, J. Shvero, R. Levy, and K. Segal, "Anaplastic carcinoma of the thyroid," European Journal of Surgical Oncology: The Journal of the European Society of Surgical Oncology and the British Association of Surgical Oncology, vol. 19, no. 6, pp. 511-516, 1993.

[9] I. Sugitani, A. Miyauchi, K. Sugino, T. Okamoto, A. Yoshida, and S. Suzuki, "Prognostic factors and treatment outcomes for anaplastic thyroid carcinoma: ATC Research Consortium of Japan cohort study of 677 patients," World Journal of Surgery, vol. 36, no. 6, pp. 1247-1254, 2012.

[10] C. Are and A. R. Shaha, "Anaplastic thyroid carcinoma: biology, pathogenesis, prognostic factors, and treatment approaches," Annals of Surgical Oncology, vol. 13, no. 4, pp. 453-464, 2006.

[11] R. Kotchetkov, J. Cinatl, A. A. Krivtchik et al., "Selective activity of BS-RNase against anaplastic thyroid cancer," Anticancer Research, vol. 21, no. 2A, pp. 1035-1042, 2001.

[12] A. Franzén and N.-E. Heldin, "BMP-7-Induced cell cycle arrest of anaplastic thyroid carcinoma cells via p21CIP1 and p27KIP1," Biochemical and Biophysical Research Communications, vol. 285, no. 3, pp. 773-781, 2001.

[13] M. V. Blagosklonny, P. Giannakakou, M. Romanova, K. B. Ain, S. E. Bates, and T. Fojo, "Effects of p53-expressing adenovirus on the chemosensitivity and differentiation of anaplastic thyroid cancer cells," The Journal of Clinical Endocrinology \& Metabolism, vol. 83, no. 7, pp. 2516-2522, 1998.

[14] Y. Nagayama, H. Yokoi, K. Takeda et al., "Adenovirus-mediated tumor Suppressorp53Gene therapy for anaplastic thyroid carcinomain vitroandin vivo," The Journal of Clinical Endocrinology \& Metabolism, vol. 85, no. 11, pp. 4081-4086, 2000.

[15] J.-Y. Jiang and F.-Y. Tseng, "Prognostic factors of anaplastic thyroid carcinoma," Journal of Endocrinological Investigation, vol. 29, no. 1, pp. 11-17, 2006.

[16] F. D. Gilliland, W. C. Hunt, D. M. Morris, and C. R. Key, "Prognostic factors for thyroid carcinoma," Cancer, vol. 79, no. 3, pp. 564-573, 1997.

[17] S. Agrawal, R. S. Rao, D. M. Parikh, H. K. Parikh, A. M. Borges, and M. B. Sampat, "Histologic trends in thyroid cancer 1969-1993: a clinico-pathologic analysis of the relative proportion of anaplastic carcinoma of the thyroid," Journal of Surgical Oncology, vol. 63, no. 4, pp. 251-255, 1996.

[18] M. Kihara, A. Miyauchi, A. Yamauchi, and H. Yokomise, "Prognostic factors of anaplastic thyroid carcinoma," Surgery Today, vol. 34, no. 5, pp. 394-398, 2004.

[19] B. Busnardo, O. Daniele, M. R. Pelizzo et al., "A multimodality therapeutic approach in anaplastic thyroid carcinoma: study on 39 patients," Journal of Endocrinological Investigation, vol. 23, no. 11, pp. 755-761, 2000.

[20] J.-P. E. N. Pierie, A. Muzikansky, R. D. Gaz, W. C. Faquin, and M. J. Ott, "The effect of surgery and radiotherapy on outcome of anaplastic thyroid carcinoma," Annals of Surgical Oncology, vol. 9, no. 1, pp. 57-64, 2002.

[21] P. I. Haigh, P. H. G. Ituarte, H. S. Wu et al., "Completely resected anaplastic thyroid carcinoma combined with adjuvant chemotherapy and irradiation is associated with prolonged survival," Cancer, vol. 91, no. 12, pp. 2335-2342, 2001.

[22] S. I. Sherma, "Thyroid carcinoma," The Lancet, vol. 361, no. 9356, pp. 501-511, 2003.

[23] J. L. Pasieka, "Anaplastic thyroid cancer," Current Opinion in Oncology, vol. 15, no. 1, pp. 78-83, 2003.

[24] T. Kobayashi, H. Asakawa, K. Umeshita et al., "Treatment of 37 patients with anaplastic carcinoma of the thyroid," Head \& Neck, vol. 18, no. 1, pp. 36-41, 1996.

[25] C.-y. Lo, K.-y. Lam, and K.-y. Wan, "Anaplastic carcinoma of the thyroid," The American Journal of Surgery, vol. 177, no. 4, pp. 337-339, 1999.

[26] I. Sugitani, N. Kasai, Y. Fujimoto, and A. Yanagisawa, "Prognostic factors and therapeutic strategy for anaplastic carcinoma of the thyroid," World Journal of Surgery, vol. 25, no. 5, pp. 617-622, 2001.

[27] S. M. Glaser, S. F. Mandish, B. S. Gill, G. K. Balasubramani, D. A. Clump, and S. Beriwal, "Anaplastic thyroid cancer: prognostic factors, patterns of care, and overall survival," Head \& Neck, vol. 38, no. S1, pp. E2083-E2090, 2016.

[28] B. McIver, I. D. Hay, D. F. Giuffrida et al., "Anaplastic thyroid carcinoma: a 50-year experience at a single institution," Surgery, vol. 130, no. 6, pp. 1028-1034, 2001.

[29] B. Roche, G. Larroumets, C. Dejax et al., "Epidemiology, clinical presentation, treatment and prognosis of a regional series of 26 anaplastic thyroid carcinomas (ATC). Comparison with the literature," Annales d'Endocrinologie, vol. 71, no. 1, pp. 38-45, 2010.

[30] T. Sato, M. Omura, J. Saito et al., "Neutrophilia associated with anaplastic carcinoma of the thyroid: production of macrophage colony-stimulating factor (M-CSF) and lnterleukin-6," Thyroid, vol. 10, no. 12, pp. 1113-1118, 2000. 
[31] T. Fujita, Y. Ogasawara, M. Naito, H. Doihara, and N. Shimizu, "Anaplastic thyroid carcinoma associated with granulocyte colony-stimulating factor: report of a case," Surgery Today, vol. 36, no. 1, pp. 63-67, 2006.

[32] K. D. Burman, M. D. Ringel, and L. Wartofsky, "Unusual types of thyroid neoplasms," Endocrinology and Metabolism Clinics of North America, vol. 25, no. 1, pp. 49-68, 1996.

[33] O. Nilsson, J. Lindeberg, J. Zedenius et al., "Anaplastic giant cell carcinoma of the thyroid gland: treatment and survival over a 25-year period," World Journal of Surgery, vol. 22, no. 7, pp. 725-730, 1998.

[34] K. B. Ain, "Anaplastic thyroid carcinoma: a therapeutic challenge," Seminars in Surgical Oncology, vol. 16, no. 1, pp. 64-69, 1999.

[35] F. Alagöl, R. Tanakol, H. Boztepe, Y. Kapran, T. Terzioğlu, and F. Dïdaroğlu, "Anaplastic thyroid cancer with transient thyrotoxicosis: case report and literature review," Thyroid, vol. 9, no. 10, pp. 1029-1032, 1999.

[36] S. Basaria, R. Udelsman, J. Tejedor-Sojo, W. H. Westra, and A. S. Krasner, "Anaplastic pseudothyroiditis," Clinical Endocrinology, vol. 56, no. 4, pp. 553-555, 2002.

[37] S. G. Francis and G. G. David, Basic \& Clinical Endocrinology, Lange Medical Book/McGraw-Hill, New York, NY, USA, 7th edition, 2004. 